sein des régions du Québec maritime qui peut se voir définir comme un cluster maritime. Reste à mettre en place, selon eux, des institutions et des mécanismes de soutien (comme le font les Brésiliens) pour accrồtre les activités du cluster en question.

On en arrive à la dernière partie, La classe créative vue sous divers angles. Ces angles sont ceux adoptés par, à nouveau, Richard Shearmur, et ensuite Guy Chiasson de l'UQO et de Myrtille (quel beau prénom, aussi beau que Gentiane...) Roy-Valex doctorante en études urbaines à l'INRS-UCS. L'espace manque pour rendre justice à ces trois auteurs qui font une analyse critique particulièrement pointue des travaux de l'icône en matière urbaine qu'est devenu Richard Florida. C'est Shearmur qui se fait le plus percutant pour, apparemment, avoir mal digéré le plat à $85 \$$ qu'il a dû avaler pour entendre le concepteur de la Classe créative et celui qui voit dans Montréal grâce, entre autres, à son index guay un grand facteur de progrès. Aurait-il influencé la décision de tenir les trop fameux outgames de juillet dernier? Shearmur estime que la question n'est pas tant de savoir s'il existe un lien entre «talent » et croissance, mais de savoir si le talent cause la croissance ou si la croissance attire le « talent ». On sait que, pour Florida, les villes doivent attirer le talent, et ensuite la croissance en découlera. Shearmur démontre ici que le contraire peut se vérifier avec une forte probabilité. De cette façon, les régions en croissance pourraient attirer les personnes éduquées faisant partie de ces $30 \%$ que Florida associe à sa classe créative. De son côté, Guy Chiasson reproche à Florida de faire peu de cas de la question de la démocratie locale qui, pourtant, fait bel et bien partie de la qualité d'un milieu de vie. En s'inspirant du cas de la Ville de Gatineau, le jeune sociologue s'interroge à savoir si une démocratie locale forte fait partie de ces ingrédients qui contribuent à rendre un milieu attrayant.

Enfin, moins critique que son professeur, M. RoyValex aborde la problématique «floridienne » en situant les rapports entre le « culturel» et «l'économique ». Elle reconnaît à l'expert d'outre frontière le mérite d'avoir su attirer l'attention des élites locales et des décideurs sur la part du culturel dans les réalités économiques nouvelles des sociétés hautement industrialisées et urbanisées.

L'ouvrage se termine par une conclusion d'ensemble due à Jean-Marc Fontan de l'UQAM. Ce grand pro- moteur de l'économie sociale devant l'éternel parvient à faire un lien entre son sujet de prédilection et l'économie des connaissances. Hé oui, qui sait ? Peutêtre que le jour n'est pas loin où les entreprises d'économie sociale d'aide domestique utiliseront des robots qui sauront repasser, faire la vaisselle et donner le bain à leurs clients en perte d'autonomie. Avec ce clin d'œil d'un humour trop facile, je ne peux que recommander fortement la lecture de ce volume dans lequel, faut-il le souligner, malgré certains auteurs que l'on invite à contribuer parce qu'ils ont bien voulu participer au colloque à l'origine du volume, on trouve véritablement ce fil conducteur, d'un riche contenu, qui fait défaut à trop d'ouvrages collectifs.

\section{Louis-Edmond Hamelin L'âme de la terre : parcours d'un géographe Québec, Éditions Multimondes 2006, 246 p.}

Quel aurait été le parcours de Louis-Edmond Hamelin s'il était né en 1973 plutôt qu'en. 1923 ? Alors qu'on ne cesse d'évoquer les problèmes qu'entraîne le réchauffement global, celui qui fut mon recteur (19771983) se serait-il rendu célèbre dans le milieu académique vers 2003 en inventant le concept de nordicité en tant que spécialiste de la géomorphologie périglaciaire ? Question sans réponse. Au début des années 1950, en terre québécoise, par l'étude des formes de terrain mises en place ou modifiées par les processus géologiques se manifestant dans un contexte de climat froid, l'un de nos plus célèbres disciples de Strabon (géographe grec ayant vécu au temps du Christ) faisait office de pionnier. Dans ce passionnant ouvrage qui prend la forme de mémoires écrites dans plusieurs chapitres à la troisième personne, Hamelin fait connaître ses faits et gestes d'une carrière académique amorcée à son retour de Grenoble avec doctorat d'université sous le bras en acceptant une poste de chargé de cours de son alma mater, l'Université Laval. Un poste qui ne tardera pas à se transformer en celui de professeur régulier qui le conduira à participer à la fondation du Centre des études nordiques.

Comment réagit-il, lui qui se distingua par des missions à travers le monde et par la réception de multiples prix, à l'inquiétude que soulève la fonte de la calotte polaire? On n'en sait rien par cet ouvrage qui décrit, entre autres choses, le Nord tel qu'il l'a connu 
à travers ses travaux à l'époque où les ours polaires s'en donnaient à cœur joie sur une banquise encore bien solide. Partagé en quatre parties (Le temps des choses, Métiers, Valeurs et Jalons historiques), l'ouvrage comprend 28 chapitres dont certains, très courts, ne font guère plus qu'une page et qui peuvent se lire dans un ordre quelconque. Entre autres, je signale : la Révolution tranquille, la géographie, la nordicité, l'autochtonie, l'écriture, le rectorat perçu et vécu (que liront avec grand intérêt les employés, anciens et actuels, de l'UQTR), la nature de l'homme. Et, à une époque où l'on reproche aux Québécois leur immobilisme, je prends plaisir à souligner le chapitre 27 intitulé : Le mobilisme. Non, il n'est pas question ici de mégaprojets auxquels les groupes de pression habituels ont pris plaisir à s'opposer. Il s'agit, on l'aura deviné, de la mobilité de l'auteur déjà qualifié « d'intellectuel vagabond ». Dans le style que l'on retrouve à travers l'ensemble de l'ouvrage, notre géographe-linguiste écrit que bouger, c'est un peu imiter le pêcheur qui, périodiquement, rejoint ses lignes appâtées. Ainsi fait-il observer que son mobilisme s'est accompagné d'un travail de défrichement dans différents domaines tels ceux du périglaciaire, de la nordologie, de l'autochtonie et de la terminologie (l'ouvrage comprend quelques dizaines de mots issus de l'imagination fertile de son auteur).

Le chapitre sur la nordicité s'avère, il va sans dire, l'un des plus importants. Le concept de nordicité (État du Nord) mis de l'avant recouvre trois aspects : une chose, un langage et des activités. Notre géographe épris de linguistique signale que le concept déborde le phénomène du froid, lequel ne constitue que l'un des facteurs nordicistes. À ses yeux, la nordicité force à concevoir le Nord autrement que les Blancs l'ont toujours fait. Ainsi, le concept conduit à « faire du Nord» dans l'esprit du Nord, ce qui oblige la reconnaissance de plain-pied des Premières Nations. On comprendra ainsi que le chapitre 15 sur les Autochtones représente un autres des chapitres dominants de l'ouvrage.

Les lecteurs de ma génération liront avec un intérêt particulier le chapitre sur la France des années 1950. $\mathrm{Au}$ lendemain de la guerre, c'est par «pleins bateaux » que de jeunes diplômés en sciences sociales ou humaines débarquent au Havre pour poursuivre leurs études à Paris et, de préférence, en province, car déjà à l'époque vivre dans la ville Lumières n'était pas sans présenter des difficultés dont étaient dépour- vues des villes de province comme Bordeaux, Strasbourg, Aix-en Provence, Dijon, Montpellier et, bien sûr, Grenoble. Dans une note de bas de page, parmi tous les noms mentionnés, on trouve: Gérard Bergeron, Léon Dion, Jean-Luc Pépin, Jeanne et Maurice Sauvé, Arthur Tremblay, Jacques Languirand, André Raynaud, Jacques Henripin. Quelque vingt ans plus tard suivront, en plus de beaucoup d'autres, l'auteur de ces lignes et le directeur d'O\&T... Ce premier séjour au pays des ancêtres fournit à Hamelin une double occasion de réfléchir aux traits du discours. Inutile de signaler que le langage qu'il entend et qu'il parle diffère sur plusieurs plans : accent tonique, accord des verbes pronominaux, et vocabulaire. En effet, combien de fois il a dû endurer l'insupportable : «Comme vous avez un accent charmant! » Oui, il appellera un bicycle une bicyclette et quand il sera tanné il dira qu'il en a marre, et au lieu de dire qu'il a dû barrer une porte, il dira qu'il l'a fermée à clé. Oui, c'est bien connu, surtout à cette époque, les Français avaient plus de vocabulaire que les Québécois. On peut se demander si ce constat n'a pas influencé Hamelin à pallier nos insuffisances langagières en relevant le défi d'inventer mots et concepts.

Pour ce qui est des mots issus de son imagination, le volume en comprend quelques dizaines, dont certains sont des trouvailles heureuses et d'autres, à mon avis, un peu moins. Un demi-siècle plus tard, Hamelin a bien raison d'écrire qu'en matière de parler, la mèrepatrie a pris nuances et couleurs. En fait, pas uniquement dans la façon de parler...

Pour terminer cette présentation qui risque fort de ne pas rendre justice à l'auteur tant le tout est riche en observations et réflexions, je me permets de signaler quelques passages qui collent bien à sa personnalité. Aimer le travail, c'est croire à ce qu'on fait, en ne se gênant pas d'y montrer la passion (Chapitre 23: Le travail). La démarche positive conduit à une hardiesse d'entreprendre et se traduit par des propositions parfois innovantes : mettre des mots peu connus, des sens inconnus ou des bravades philosophiques dans des titres d'ouvrage. (Chapitre 25: Confiance en soi). Marcher, c'est mettre en arrière de soi la partie du monde qui apparaît devant soi (Chapitre 23: Le travail). Le devenir de l'après-décès soulève une grande inconnue à la fois pour l'âme et pour le corps, mais les religions, les philosophes et les sciences anticipent des cheminements. Mieux vaut être serein qu'affolé. (Postface: De la relativité de la personne). Un livre 
émouvant qui intéressera tous les lecteurs de d'O\&T de part et d'autre de l'Atlantique.

\section{Roméo Bourchard \\ Y a t-il un avenir pour les régions? Un projet d'occupation du territoire Montréal, Écosociété, 2006, 224 p.}

L'intérêt du sujet et celui que suscite toujours son coloré auteur ont fait de cet ouvrage un événement attendu. Le Devoir en avait prévenu ses lecteurs deux semaines avant le lancement. C'est tout dire. Oui, de la part du médiatique fondateur de l'Union paysanne, il y avait lieu de s'attendre à un texte percutant. Les plus fidèles supporteurs de celui que s'est fait le chevalier défenseur de la ferme familiale de dimension humaine ne devraient pas être déçus. On l'aura deviné, il est très peu question de Montréal et de Québec dans ce volume. Quand on parle de régions au Québec, l'usage veut qu'on se rapporte aux territoires autres que ceux appartenant à la métropole ou à la capitale nationale. Et comme on ne peut évoquer le mot région sans penser au déclin qui affecte bon nombre d'entre elles, à savoir les régions ressources dites aussi périphériques, c'est surtout vers elles que l'auteur consacre son attention.

Un premier très court chapitre, où il est fait allusion aux melons (!) de Montréal (oui, on en trouve d'excellents aux marchés Jean-Talon et Atwater) permet à l'auteur de jeter ses cartes en reprenant à son compte le célèbre dicton Deux Québec dans un. À mon avis, Trois ou quatre Québec dans un, comme pour tout pays, conviendrait mieux pour traduire notre réalité, mais passons. Oui, passons aussi rapidement sur un chapitre 2 qui, chiffres à l'appui, évoque le déclin des régions (enfin pas celles de la Montérégie ou des Basses-Laurentides ou encore de Lanaudière). On le sait, oublions Huxley, tout ne va pas pour le mieux dans le meilleur des mondes pour nos régions éloignées des grands centres. Pôvre Gaspésie, en effet! Et que dire du Saguenay-Lac-Saint-Jean avec son autocar hebdomadaire rempli de jeunes nantis d'un billet « aller ».

Passons aussi au-delà des chapitres 3 et 4 qui ne font que de la figuration, non sans contenir quelques affirmations gratuites et parfois fort contestables (cf. les affirmations sur la Politique nationale de la ruralité qui ne tiennent pas la route) et allons directement au cœur du volume, où cette fois la critique se veut de toute évidence constructive. Son titre est bien choisi : Un projet d'occupation $d u$ territoire. Après avoir épinglé dans le chapitre précédent la pratique du développement local qu'il considère rien de moins qu'un échec, ici Bouchard se fait plus conciliant et recommande (sans mentionner les CLD et les SADC) d'utiliser tout ce que le développement local a mis en place pour reconstruire des économies régionales et faire de la marginalité un atout plutôt qu'un handicap. Mais, pour y arriver, il faut des pouvoirs. Oui, des pouvoirs dont doivent être investies les MRC. Pour ne pas avoir trois ou quatre Québec en un, il importe de donner une voix politique aux régions. Pour notre militant agriculteur biologique, ça veut dire que les MRC doivent recevoir le statut de gouvernement territorial local. En effet, nous n'allons pas le contredire lorsqu'il écrit que c'est la MRC et non la région qui correspond à l'unité territoriale et aux communautés d'appartenance de base. Alors, aux urnes citoyens! Élisons préfets et conseils exécutifs. Et qu'on cesse de parler de décentralisation et qu'on la réalise effectivement.

Sans tomber dans les sempiternels «Faukon» et «Yaka » («faut qu'on » fasse ceci et «y a » qu'à faire cela), Bouchard avance des propositions qui, pour employer son langage, relèvent du gros bons sens. Deux exemples : favoriser les productions d'appellation (dada de Solidarité rurale du Québec); partager les redevances générées par les ressources naturelles. En relation avec cette dernière proposition, le lecteur lira avec grand intérêt les réflexions sur la remise en valeur des secteurs d'activités qui ont fait le Québec : la forêt, les mines, la pêche, l'agriculture (oui, malgré la concentration outrancière, tout espoir n'est pas perdu), l'énergie, l'eau. À ceux-ci s'ajoutent de nos jours le récrétourisme : une véritable mine d'or si on y porte attention. Comment ne pas être d'accord avec l'auteur quand il souligne l'importance de développer des créneaux et des positionnements originaux. Ceci, oui, en veillant à la qualité professionnelle des produits offerts.

Ce stimulant chapitre se poursuit en insistant sur la décentralisation des services dans les domaines de l'éducation, de la santé et services sociaux, le transport et enfin la culture (ne craignez rien, il n'est pas recommandé de démanteler les ministères s'y rapportant). On y trouve même un préjugé favorable envers la contribution de l'économie sociale (avec preuves à 